\title{
Spin interferometry in anisotropic spin-orbit fields
}

\author{
Henri Saarikoski, ${ }^{1}$ Andrés Reynoso,${ }^{2,3}$ José Pablo Baltanás, ${ }^{4}$ Diego Frustaglia, ${ }^{4}$ and Junsaku Nitta ${ }^{5}$ \\ ${ }^{1}$ RIKEN Center for Emergent Matter Science (CEMS), \\ 2-1 Hirosawa, Wako, Saitama 351-0198, Japar ${ }^{*}$ \\ ${ }^{2}$ Instituto Balseiro and Centro Atómico Bariloche, \\ Comisión Nacional de Energía Atómica, 8400 Bariloche, Argentina \\ ${ }^{3}$ Consejo Nacional de Investigaciones Científicas y Técnicas (CONICET), Argentin ${ }^{\dagger}$ \\ ${ }^{4}$ Departamento de Fisica Aplicada II, Universidad de Sevilla, E-41012 Sevilla, Spain and \\ ${ }^{5}$ Department of Materials Science, Tohoku University, Sendai 980-8579, Japan
}

(Dated: November 14, 2018)

\begin{abstract}
Electron spins in a two-dimensional electron gas (2DEG) can be manipulated by spin-orbit (SO) fields originating from either Rashba or Dresselhaus interactions with independent isotropic characteristics. Together, though, they produce anisotropic SO fields with consequences on quantum transport through spin interference. Here we study the transport properties of modelled mesoscopic rings subject to Rashba and Dresselhaus [001] SO couplings in the presence of an additional inplane Zeeman field acting as a probe. By means of $1 \mathrm{D}$ and $2 \mathrm{D}$ quantum transport simulations we show that this setting presents anisotropies in the quantum resistance as a function of the Zeeman field direction. Moreover, the anisotropic resistance can be tuned by the Rashba strength up to the point to invert its response to the Zeeman field. We also find that a topological transition in the field texture that is associated with a geometric phase switching is imprinted in the anisotropy pattern. We conclude that resistance anisotropy measurements can reveal signatures of SO textures and geometric phases in spin carriers.
\end{abstract}

\section{INTRODUCTION}

The electron spin couples directly to magnetic fields via the Zeeman effect and indirectly to electric fields via the spin-orbit $(\mathrm{SO})$ interaction, that is due to a special relativistic effect: an electric field in the laboratory frame gets a magnetic component in the moving electron's rest frame due to relativistic corrections, which couples to the electron spin. In mesoscopic quantum wells made of III-V zinc-blende semiconductors two different types of SO interaction arise. The asymmetric potential of the quantum well gives rise to the Bychkov-Rashba SO interaction via structure inversion asymmetry ${ }^{1}$ In addition, bulk inversion asymmetry of the crystal structure leads to the Dresselhaus SO interaction? The magnitude of the SO fields may be strong, so that effective fields of several teslas can be generated in semiconductors, which provides effective ways to manipulate spin. Moreover, the Rashba SO interaction can be tuned with a gate electrode ${ }^{3}$ These properties have made SO fields especially relevant in studies of spin phenomena.

For instance, the combination of Rashba and Dresselhaus SO interactions can be used to design complex spin textures. ${ }^{[4}$ In this way, when the linear Rashba and Dresselhaus terms equal each other, an helical spin density wave (persistent spin helix state) emerges, allowing a huge increase in spin lifetime ${ }^{5}$ due to the spin protection by $\mathrm{SU}(2)$ symmetry ${ }^{6}$ Moreover, the possibility of an electric tuning of the Rashba interaction opens a door to an efficient control of such spin helix state ${ }^{7}$ with potential applications for nonballistic spin transistors. ${ }^{8}$ However, realistic implementations are hindered by the complexity of SO fields in semiconductor heterostructures. Besides, applications to several spin phenomena and SO-induced spin structures require an accurate prediction and in-situ determination of the SO interaction parameters $\frac{9110}{}$.

A different prominent feature resulting from the combination of Rashba and Dresselhaus SO fields in a 2D electron gas (2DEG) is the anisotropic character of electron transport ${ }^{11}$ The spin-orbit fields in a 2DEG are associated with weak localization/antilocalization effects. 12 For example, it is known that an in-plane magnetic field induces a characteristic resistance anisotropy caused by weak localization! ${ }^{[3]}$ Complex spin-orbit fields in combination with an in-plane magnetic field are also expected to cause resistance anisotropy in mesoscopic ring systems due to the influence of circular interference paths.

In this work, we study electron spin interferometry in mesoscopic rings subject to $k$-linear Rashba and Dresselhaus SO interactions in the presence of an external magnetic field applied within the plane of electron transport. The most notable feature emerging in our calculations is anisotropy in the resistance as a function of the in-plane magnetic field direction. In contrast to the 2DEG this anisotropy is associated with the spin interference around the ring under the influence of spinorbit fields 14 As shown below, time-reversed symmetric Altshuler-Aronov-Spivak (AAS $)^{15}$ paths play a crucial role in the effect in moderate disorder densities. We predict oscillations in the resistance anisotropy as a function of both the in-plane and Rashba fields strengths. Furthermore, we demonstrate signatures of an effective geometric phase switching in transport anisotropy.

\section{THE MESOSCOPIC RING SYSTEM}

Our system consists of a narrow straight mesoscopic wire of width $W$ connected symmetrically to a ring of 


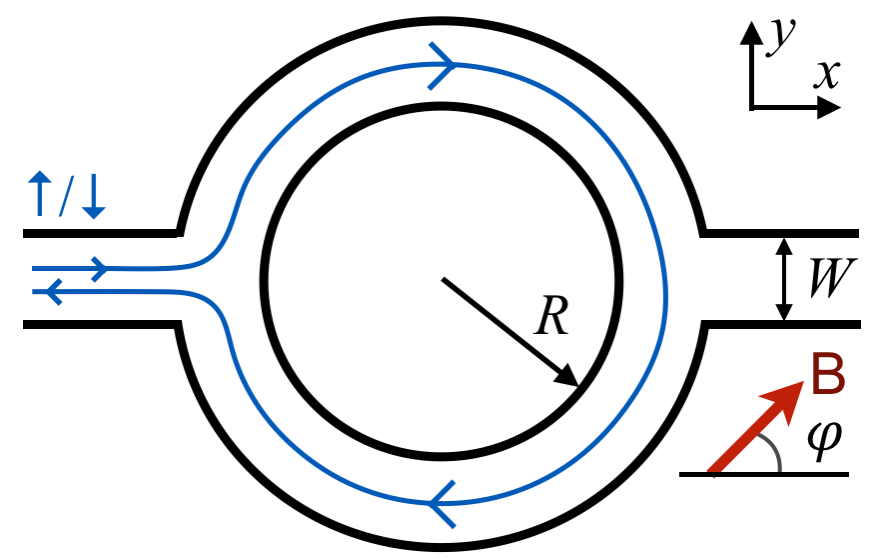

FIG. 1. Geometry of the 2D ring system. The blue line shows the clockwise propagating Altshuler-Aronov-Spivak interference path for spins $\uparrow, \downarrow$ that we use in the 1D models. The time-reversed counterpart is propagating in the opposite direction.

inner radius $R$ and the same width, containing a 2DEG. The geometry of the system is depicted in Fig. 1. The lead orientation angle with respect to the $x$-axis is denoted by $\omega$. We keep $\omega=0$ in the calculations, as in Fig. [1. except when otherwise stated.

Structure and bulk inversion asymmetry results in the appearance of SO coupling terms. In particular, the linear Bychkov-Rashba SO interaction may be written as 1

$$
H_{\mathrm{R}}=\alpha\left(k_{y} \sigma_{x}-k_{x} \sigma_{y}\right),
$$

where $\mathbf{k}=k_{x} \hat{\mathbf{x}}+k_{y} \hat{\mathbf{y}}+k_{z} \hat{\mathbf{z}}$ is the electron $k$-vector and $\sigma_{x, y, z}$ denote Pauli matrices. In turn, the Dresselhaus SO interaction depends on the crystal axis orientation in the quantum well. We consider here the high-symmetry [001] orientation and neglect $k$-cubic terms. This leads to $16 \mid 17$

$$
H_{\mathrm{D},[001]}=\beta\left(k_{y} \sigma_{y}-k_{x} \sigma_{x}\right) .
$$

Here, we are assuming that $\alpha, \beta>0$. Besides, we consider an in-plane magnetic field

$$
\mathbf{B}(\varphi)=B(\cos \varphi \hat{\mathbf{x}}+\sin \varphi \hat{\mathbf{y}})
$$

with $\varphi$ an arbitrary angle characterizing the field direction with respect to the wire axis (see Fig. 1). This results in the Zeeman term

$$
H_{\text {Zeeman }}=g^{*} \mu_{\mathrm{B}} \mathbf{B} \cdot \boldsymbol{\sigma},
$$

where $\boldsymbol{\sigma}=\sigma_{x} \hat{\mathbf{x}}+\sigma_{y} \hat{\mathbf{y}}+\sigma_{z} \hat{\mathbf{z}}$ is the vector of Pauli matrices, $\mu_{\mathrm{B}}$ is the Bohr magneton and $g^{*}$ is the effective $g$-factor.

After including the kinetic term, the full Hamiltonian reads

$$
H=\hbar^{2} k^{2} / 2 m^{*}+H_{\mathrm{R}}+H_{\mathrm{D},[001]}+H_{\text {Zeeman }},
$$

where $m^{*}$ is the effective mass.
The combination of Rashba and Dresselhaus SO interactions leads to an anisotropic spin energy in $k$ space $^{18}$ (Fig. 2 a) experienced by the spin carriers as an anisotropic internal magnetic field with extreme values $|\alpha-\beta| k$ and $|\alpha+\beta| k$ along the directions $(1,1) / \sqrt{2}$ and $(1,-1) / \sqrt{2}$ in $k$-space, respectively. The anisotropic spin energy can be probed by introducing an external Zeeman field coplanar with the ring, giving rise to anisotropic transport effects with respect to the Zeeman field direction $\varphi$.

\section{1D AND 2D METHODS}

An appropriate treatment of multiple-mode transport in the wire requires the use of full 2D simulations. Here, we use the Kwant code ${ }^{19}$ to implement a tight-binding type Hamiltonian in a 2D grid and calculate the conductance through the system. We use values of the material parameters compatible with those of InGaAs, with $m^{*}=0.05 m_{0}$, where $m_{0}$ is the bare electron mass, and $g^{*}=3$. An in-plane field strength of $B=1 \mathrm{~T}$ corresponds then to $0.17 \mathrm{meV}$. We keep the Fermi energy at $47.7 \mathrm{meV}$ in all the calculations, which gives an electron density of approximately $n=1.0 \times 10^{12} / \mathrm{cm}^{2}$ in the 2 DEG at Fermi wavenumber $k_{\mathrm{F}}=2.46 \times 10^{8} / \mathrm{m}$. The ring inner radius $R$ is fixed to $610 \mathrm{~nm}$, while the wire width $W$ is adjusted between $42 \mathrm{~nm}$ and $68 \mathrm{~nm}$, the former giving 3 conducting modes and the latter giving 5 of them.

Disorder is introduced in the system by adding a spinindependent random scattering potential in the lattice.20 The resulting conductance is strongly dependent on the particular realization of this random potential, hence we perform disorder averaging in our simulations, giving the statistical accuracy of our results in terms of $\pm \sigma$ errorbars. In experiments, this may be done effectively by constructing a self-averaging sample consisting in a network containing a large number of rings. 21 . In this setup, AAS paths are especially important ${ }^{22}$ since direct interference paths are strongly affected by small sample asymmetries, disorder, scattering between the transport modes as well as energy averaging. For AAS time-reversed symmetric paths, on the contrary, the acquired phase $k_{F} 2 \pi R$ between clockwise and anticlockwise winding paths is cancelled out (in contrast to the spin phase difference), so the effects of disorder are minimized. With this in mind, we use a disorder mean-free path of $\ell=2.5 \mu \mathrm{m}$ unless otherwise stated. This is shorter than the inner circumference of the ring, $3.8 \mu \mathrm{m}$, and comparable to the estimated mean free path in the array of semiconductor rings in Ref. 21. This moderate disorder density favours the AAS interference paths (see Fig. 1) prevailing in the experiments.

In addition, we apply approximate 1D models to gain physical insights into the spin dynamics and, in particular, into the origin of the anisotropy in resistance reported below. We neglect curvature and torsion effects that arise due to the steep curvature at the intersection. ${ }^{2324}$ On 

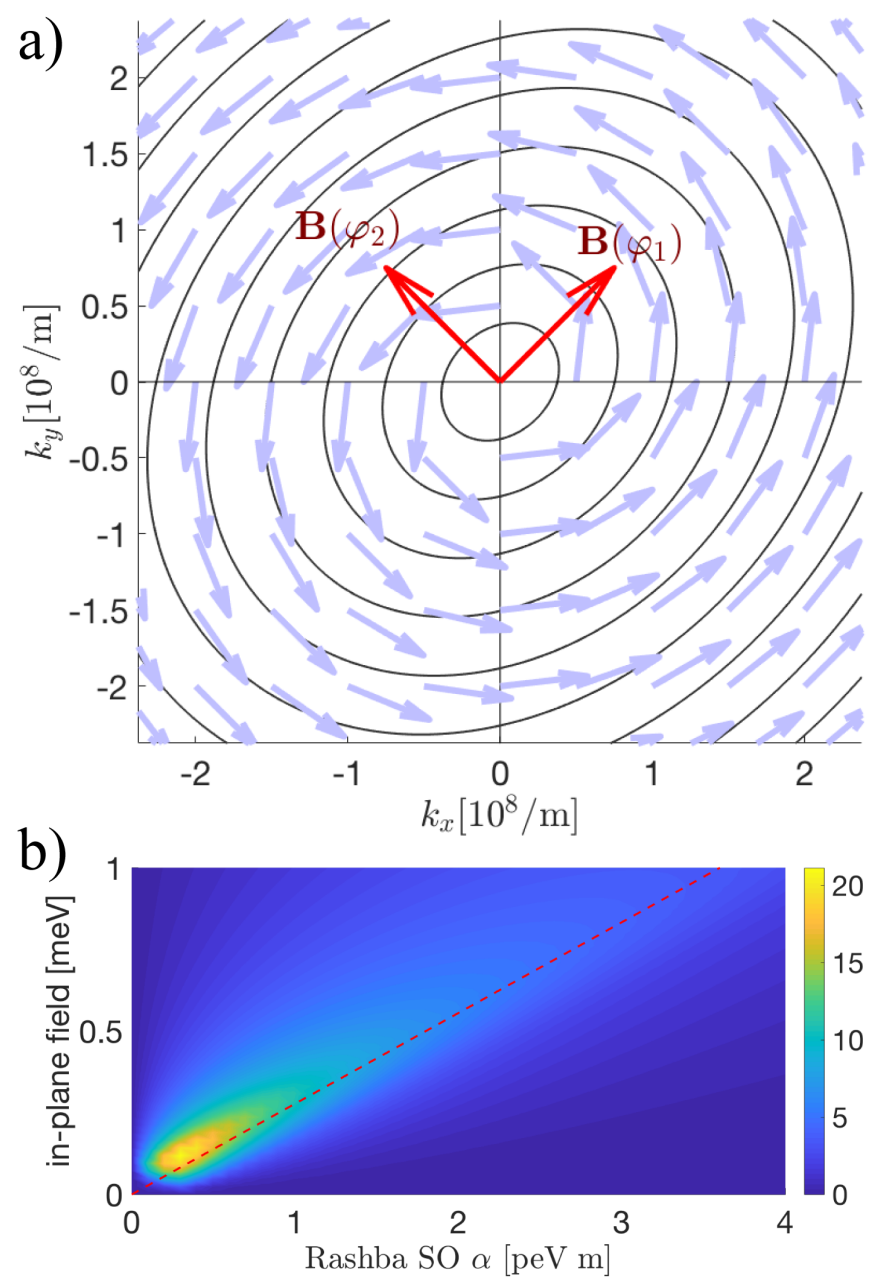

FIG. 2. a) The combination of Rashba and Dresselhaus spinorbit interactions leads to an anisotropic spin-orbit energy in $k$-space. Spin-orbit parameters are here $\alpha=2.64 \mathrm{peV} \mathrm{m}$ and $\beta=0.3 \mathrm{peV} \mathrm{m}$. The figure shows energy countours of the spin Hamiltonian (solid lines) and directions of the spin eigenstates (arrows). Angles $\varphi_{1}=\pi / 4$ and $\varphi_{2}=3 \pi / 4$ correspond to the extreme values of the spin orbit field in the ring. b) Anisotropy (in \%) of the total average effective field $B_{\text {ave }}(\varphi)[$ Eq. $(9]$ ] acting on a spin along a circular path around the ring in the 1D spin-rotation model. Anisotropy is calculated from $\left(B_{\text {ave }}(\varphi=\pi / 4)-B_{\text {ave }}(\varphi=3 \pi / 4)\right) /\left[B_{\text {ave }}(\varphi=\right.$ $\left.\pi / 4)+B_{\text {ave }}(\varphi=3 \pi / 4)\right] / 2$, as a function of the Rashba and in-plane field strengths. The Dresselhaus interaction energy is fixed to $\beta=0.3 \mathrm{peV} \mathrm{m}$ and a ring radius $R=610 \mathrm{~nm}$ is used. The dashed line shows where the Rashba SO field strength is equal to the in-plane field strength.

the one hand, we use a 1D tight-binding-based approach that incorporates the full Hamiltonian in a chain (see Appendix A 1 for details). On the other hand, we introduce a 1D spin-rotation model that neglects the orbital part of the Hamiltonian. In this model we assume moderate disorder density in the system and focus therefore on the AAS interference paths. Thus, the total probability of backscattering is calculated from the reflected wave at the intersection and waves transmitted around the ring from

$$
\psi=p_{1} \psi_{0}+p_{2}\left(\psi_{+}+\psi_{-}\right)
$$

where the spinor states $\psi_{+,-}$correspond to clockwise $(+)$ and anticlockwise (-) moving spins, $\psi_{0}$ stands for directly reflected spins, and $p_{1,2}$ are the backscattering amplitudes for directly reflected spins and spins propagating once around the ring, respectively. As shown in Appendix A 2, the resistance can be expressed in a closed form in terms of $\psi$.

In the following, we use two independent characterizations of the anisotropy observed in the resistance $R_{\Omega}(\varphi)$ across the ring. Resistance anisotropy between fixed axes corresponding to different in-plane orientations $\varphi_{A}$ and $\varphi_{B}$ is calculated from

$$
A_{\mathrm{R}}\left(\varphi_{A}, \varphi_{B}\right)=\frac{R_{\Omega}\left(\varphi_{A}\right)-R_{\Omega}\left(\varphi_{B}\right)}{\left[R_{\Omega}\left(\varphi_{A}\right)+R_{\Omega}\left(\varphi_{B}\right)\right] / 2}
$$

by using the methods described above. In addition, when possible, we compute the angle-averaged resistance $\left\langle R_{\Omega}(\varphi)\right\rangle=\int_{0}^{\pi} R_{\Omega}(\varphi) \mathrm{d} \varphi / \pi$, from which we calculate the $\varphi$-dependent resistance anisotropy

$$
A_{\mathrm{R}}(\varphi)=\frac{R_{\Omega}(\varphi)-\left\langle R_{\Omega}(\varphi)\right\rangle}{\left\langle R_{\Omega}(\varphi)\right\rangle}
$$

Resistance is given here in units of $1 / G_{0}$, where $G_{0}=$ $e^{2} / h$ is the quantum of conductance.

\section{TRANSPORT ANISOTROPY}

In the following we focus on anisotropic SO fields 25 where the Dresselhaus SO interaction is low, i.e., $\beta \ll \alpha$. We consider first the case of zero in-plane magnetic field, $\mathbf{B}=0$. Aharonov-Casher resistance oscillations calculated as a function of the Rashba spin-orbit coupling $\alpha$ in a disordered 3-mode ring system are shown in Fig. 3a for a fixed Dresselhaus SO coupling $\beta=0.3 \mathrm{peV}$, close to the Dresselhaus interaction strength found in InGaAs heterostructures. $\frac{10}{10}$ We point out that the oscillation frequency of the resistance as a function of $\alpha$ is twice the frequency of the oscillations due to direct interference paths, which become dominant in the clean limit of ballistic transport. This allows us to associate the observed behavior with AAS interference paths. In fact, the results are adequately reproduced by the 1D single-mode spin-rotation model with AAS paths for an appropriate choice of the scatterig amplitudes $\left(p_{1}=0, p_{2}=0.12\right)$.

We add now an external in-plane magnetic field to the SO field. The average effective field strength acting on a spin in a round-trip around the ring becomes then anisotropic. This can be illustrated by computing the magnitude of the average effective field for a onedimensional single-channel ring as a function of $\varphi$ (see Appendix A 2

$$
B_{\text {ave }}(\varphi)=\int_{0}^{2 \pi}\left|\mathbf{B}_{\mathrm{R}}+\mathbf{B}_{\mathrm{D}}+g^{*} \mu_{\mathrm{B}} \mathbf{B}_{\text {in-plane }}(\varphi)\right| d \theta / 2 \pi
$$


and comparing its values for two different orientations of the in-plain field. This is depicted in Fig. 2b. Notice that the field anisotropy is largest when all the field components are of comparable magnitude.

The anisotropic nature of the effective field manifests as a anisotropy in the resistance across the ring. In particular, for fixed Rashba, Fig. 3 3 shows a non-zero resistance anisotropy $A_{\mathrm{R}}(\varphi)$, strongly dependent on the external field orientation. Likewise, an unambiguous oscillation of the resistance anisotropy as a function of $\alpha$ is observed when the direction of the external field remains fixed. This is most visible in Figs. 4 a and b, showing the resistance anisotropy as a function of the field orientation $\varphi$ and the Rashba SO strength $\alpha$ for fixed $\beta=0.3 \mathrm{peV} \mathrm{m}$, calculated using 2D simulations and the $1 \mathrm{D}$ spin-rotation model. Degree of anisotropy decreases with increasing $\alpha$ since the field anisotropy also decreases (Fig. $2 \mathrm{~b}$ ).

Figures 4 and b show a change in the sign of phase of resistance anisotropy at $\alpha=2.1 \mathrm{peV} \mathrm{m}$ and again at about $3.3 \mathrm{peVm}$. Using definition (7) this is described by the oscillating sign of $A_{\mathrm{R}}(\varphi=\pi / 4, \varphi=3 \pi / 4)$ as a function of $\alpha$. Figure 4 . shows oscillations in $A_{\mathrm{R}}(\varphi=$ $\pi / 4, \varphi=3 \pi / 4)$ as reproduced by the $1 \mathrm{D}$ tight-binding approach. Anistropy changes sign here at $\alpha=2.5 \mathrm{peV} \mathrm{m}$, $3.3 \mathrm{peV} \mathrm{m}$ and $3.8 \mathrm{peV} \mathrm{m}$. We note that the overall behavior in the $1 \mathrm{D}$ tight-binding approach is consistent with the $1 \mathrm{D}$ spin rotation model as well as the $2 \mathrm{D}$ calculations in Figs. 4 a and b.

In addition to resistance anisotropy originating from AAS spin interference multi-mode rings are expected to display resistance anisotropy caused by weak localization/antilocalization in the multi-mode wire.13. However, the resistance anisotropy due to the latter contribution reverses sign only when either the Rashba or Dresselhaus coupling parameter changes its sign. This is in contrast to our results that show oscillations in the sign of $A_{\mathrm{R}}(\varphi=\pi / 4, \varphi=3 \pi / 4)$ with increasing spin-orbit field (Fig. 4). We find that this pattern of anisotropy sign reversal persists at moderate disorder densities with $0.45 \mu \mathrm{m}<\ell<3.0 \mu \mathrm{m}$ (see Appendix D).

We find no sign reversal in $A_{\mathrm{R}}(\varphi=\pi / 4, \varphi=3 \pi / 4)$ in the very disordered regime $\ell<0.15 \mu \mathrm{m}$. Resistance anisotropy originates then mainly from the weak localization/antilocalization effects in the multi-mode wire (see Appendix D. On the other hand, in the regime of weak disorder $\ell>3.0 \mu \mathrm{m}$ spin interference from direct path Aharonov-Casher contributions start to dominate changing the pattern of resistance anisotropy. These calculations indicate that AAS ring interference is the most important source of resistance anisotropy in the 2D simulations at moderate disorder densities.

\section{A. Anisotropy in the ring spectrum}

To clarify the origin of the resistance anisotropy we study the eigenstates of an isolated single-mode ring. In the absence of both Dresselhaus and in-plane fields, an
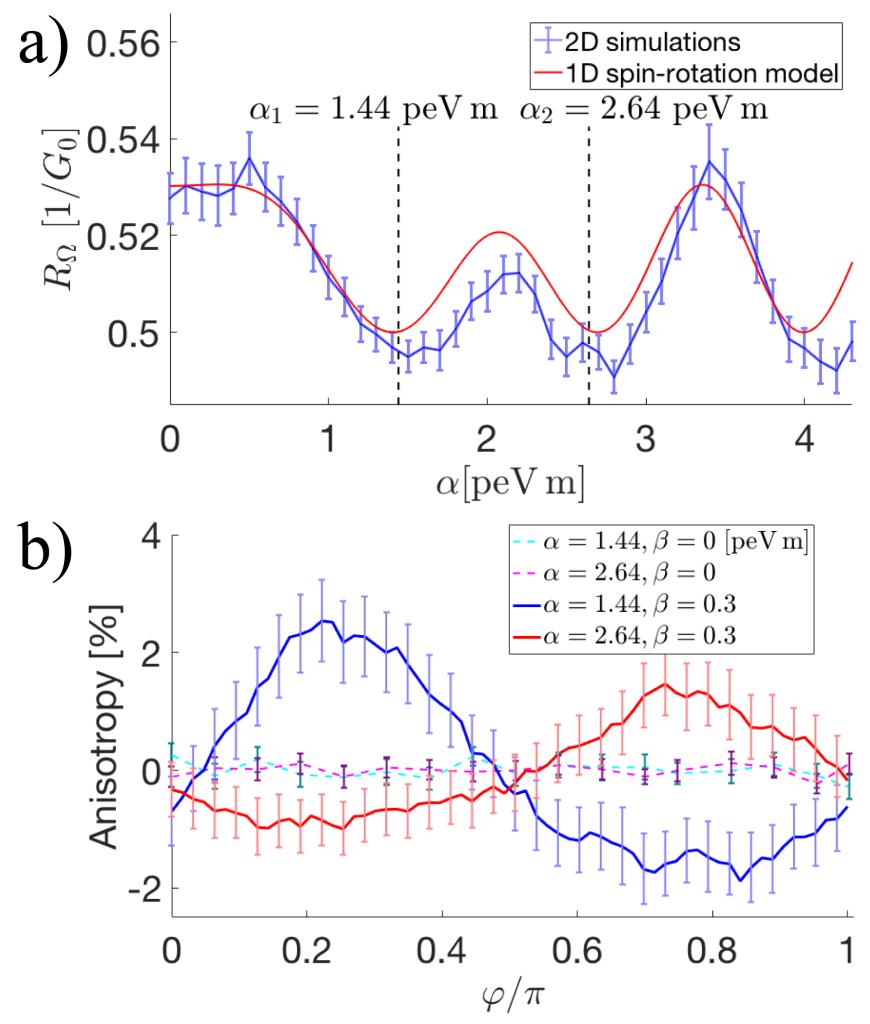

FIG. 3. a) Resistance in a 3-mode ring system as a function of Rashba SO coupling $\alpha$ and at fixed Dresselhaus SO coupling $\beta=0.3 \mathrm{peV} \mathrm{m}$ in the absence of an external magnetic field $(\mathbf{B}=0)$. The results are calculated using the $2 \mathrm{D}$ model and compared to those obtained with the singlemode 1D spin-rotation model with AAS paths $\left(p_{1}=0\right.$, $\left.p_{2}=0.12\right)$. The Rashba couplings $\alpha_{1,2}$ shown in the figure coincide approximately with the first resistance minima. b) Resistance anisotropy $A_{\mathrm{R}}(\varphi)$ [Eq. (8)] corresponding to an inplane Zeeman field strength of $0.17 \mathrm{meV}$ and Rashba SO fields $\alpha_{1}=1.44 \mathrm{peV} \mathrm{m}$ and $\alpha_{2}=2.64 \mathrm{peV} \mathrm{m}$. The Dresselhaus field strength is fixed to $\beta=0.3 \mathrm{peVm}$. For comparison, we include the case $\beta=0$ showing no anisotropy (dashed lines), see Appendix B.

exact expression for the energy levels corresponding to the different eigenstates can be found 27 , which reads

$E=\frac{\hbar^{2}}{2 m^{*} R^{2}}\left[\left(\lambda n+\frac{1}{2}\right)^{2}+\frac{1}{4}+s\left|\lambda n+\frac{1}{2}\right| \sqrt{1+\left(\frac{2 \alpha m^{*} R}{\hbar^{2}}\right)^{2}}\right]$

where $\lambda= \pm 1$ denotes the rotation direction of the electron in the ring, $s= \pm 1$ its spin, and $n>0$ is the (integer) orbital quantum number. For a vanishing SO field $(\alpha=0)$, the eigenstates show a 4-fold degeneracy: a 2-fold Kramers degeneracy associated with the rotation direction around the ring and a 2-fold spin degeneracy. Spin degeneracy is lifted by the Rashba spinorbit interaction (upper panel in Fig. 5a). Eventually, as the Rashba strength increases new level crossings show up and the 4-fold degeneracy is restored, but this time involving states of different $n, \lambda$, and $s$, which carry a different total phase. An example is shown in the 

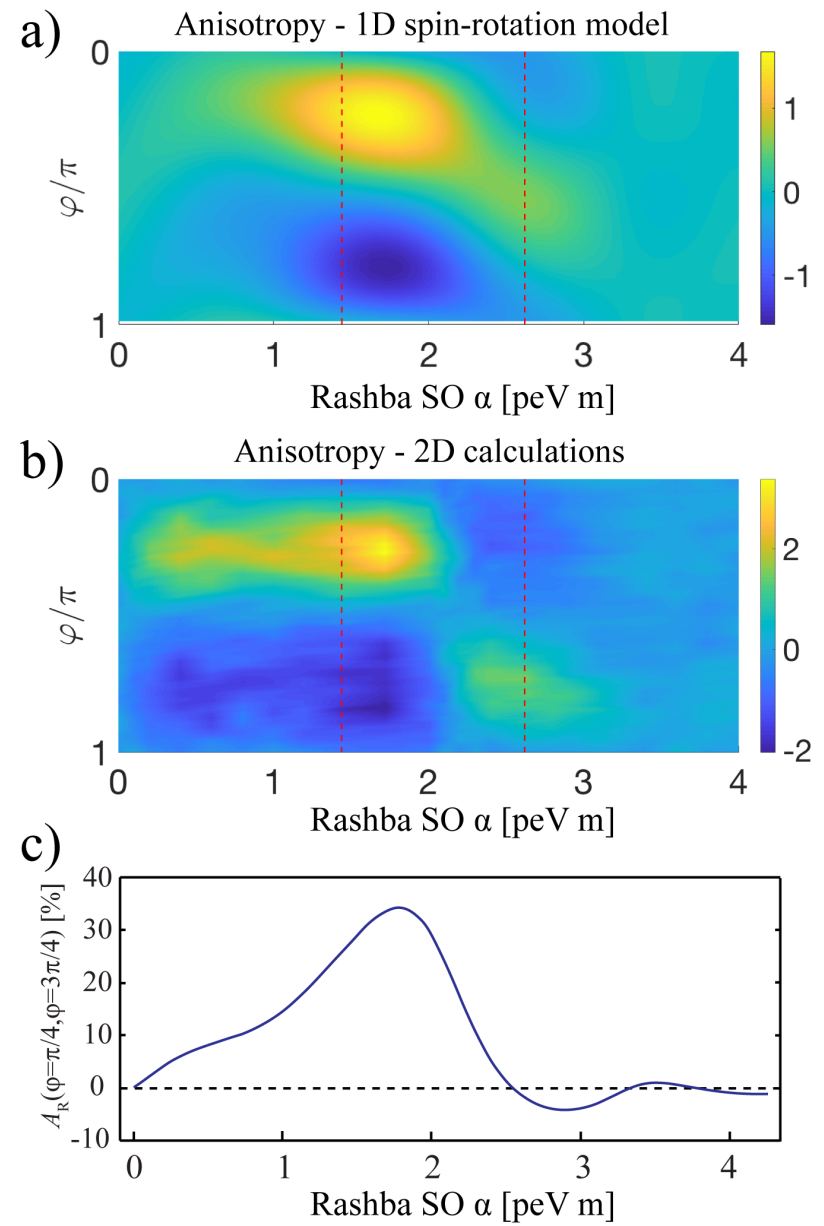

FIG. 4. Resistance anisotropy $A_{\mathrm{R}}(\varphi)$ [Eq. [8] ], in \%, for fixed Dresselhaus $(\beta=0.3 \mathrm{peVm})$ and in-plane $(0.17 \mathrm{meV})$ magnetic fields as a function of the Rashba spin-orbit coupling $\alpha$ and the in-plane field direction $\varphi$. Panels (a) and (b) correspond to the results obtained with the 1D spinrotation model and full $2 \mathrm{D}$ transport simulations, respectively. The $1 \mathrm{D}$ model uses backscattered AAS paths $\left(p_{1}=0\right.$, $p_{2}=0.12$ ). In both cases, a "phase switch" in anisotropy is observed close to $\alpha=2.1 \mathrm{peV}$. The dashes lines indicate the Rashba SO couplings $\alpha_{1,2}$ shown in Fig. 3. c) Resistance anisotropy $A_{\mathrm{R}}(\varphi=\pi / 4, \varphi=3 \pi / 4)$ calculated with the $1 \mathrm{D}$ tight-binding method demonstrates an oscillating phase in anisotropy. Anisotropy is calculated from the AAS paths with the Dresselhaus SO strength $\beta=0.3 \mathrm{peV}$ m, and in-plane field strength $0.17 \mathrm{meV}$. Isotropic background conductance in the calculation is fixed to $G_{\mathrm{B}}=2.5 G_{0}$ (see Appendix A 1).

upper panel of Fig. 5 a where a 4-fold degeneracy appears near $\alpha=2.2 \mathrm{peV} \mathrm{m}$ and $E=47.1 \mathrm{meV}$ for states corresponding to $(n=143, s=+1, \lambda=+1)$, $(n=144, s=-1, \lambda=-1),(n=145, s=-1, \lambda=+1)$, and ( $n=146, s=+1, \lambda=-1)$ with a round-trip phase difference of $2 \pi$ and $6 \pi$ for $s=-1$ and $s=+1$ spin species, respectively. This is associated with the resistance maximum for AAS transport due to constructive backscattering interference of the same spin species.

The presence of an anisotropic SO field $(\beta \neq 0)$ and
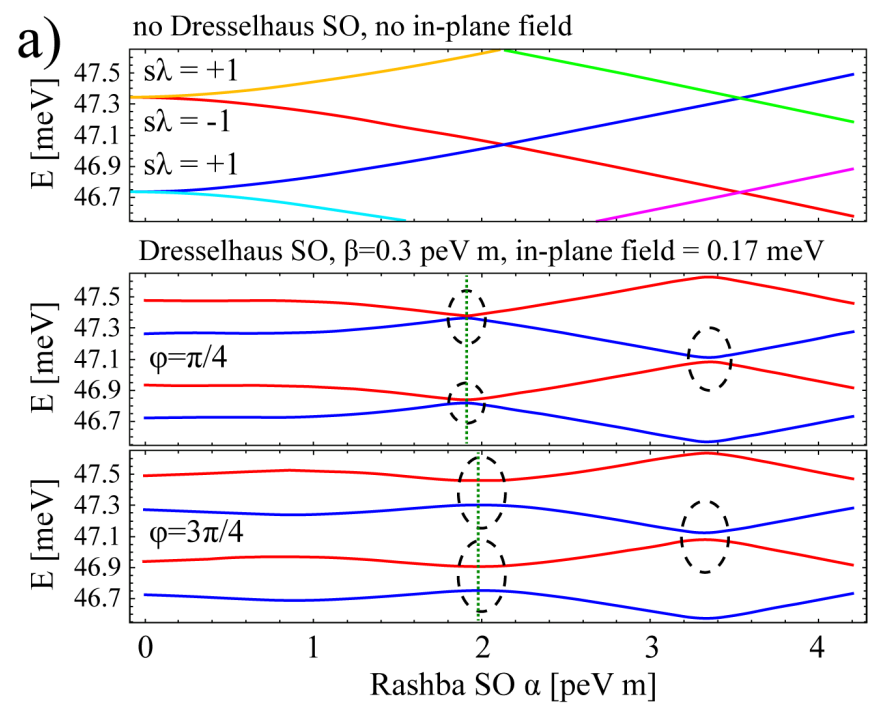

b) AAS conductance, $\beta=0.3 \mathrm{peV} \mathrm{m}$, in-plane field $=0.17 \mathrm{meV}$

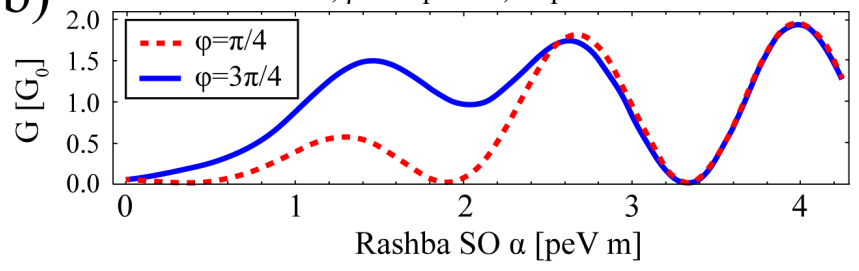

FIG. 5. a) Eigenstate spectrum of an isolated spin-orbit ring system as a function of the Rashba spin-orbit coupling $\alpha$ in the cases of vanishing Dresselhaus and in-plane fields (upper panel) and finite Dresselhaus and in-plane fields, $\beta=$ $0.3 \mathrm{peV} \mathrm{m}$ and $B=0.17 \mathrm{meV}$, for two in-plane orientations, $\varphi=3 \pi / 4$ (middle panel) and $\varphi=\pi / 4$ (lower panel). Anticrossing gaps appear in the spectrum. Gap sizes (circles) and positions (dashed green lines) depend on the in-plane field direction. The results are computed using the $1 \mathrm{D}$ tight-binding model. b) Corresponding AAS anisotropic conductance at $\varphi=\pi / 4$ and $\varphi=3 \pi / 4$.

an in-plane Zeeman field modifies the previous description substantially. In particular, anti-crossing gaps open (dashed lines in the lower panel of Fig. 5a). This is detrimental to the constructive interference of the AAS paths and, consequently, the conductance at the gap position increases. Most importantly, both the gap sizes and positions depend on the in-plane field direction, from which an anisotropy in conductance naturally emerges. This is demonstrated in Fig. 5b for two different orientations of the Zeeman field $\varphi=\pi / 4$ and $\varphi=3 \pi / 4$ : The larger gap sizes appearing at slightly different values of $\alpha$ for $\varphi=3 \pi / 4$ result in a higher AAS conductance with shifted minima when compared with the case $\alpha=\pi / 4$. In addition, the sizes of the gaps appearing in the anisotropic spectrum decreases with $\alpha$ and, correspondingly, so does the anisotropy. 


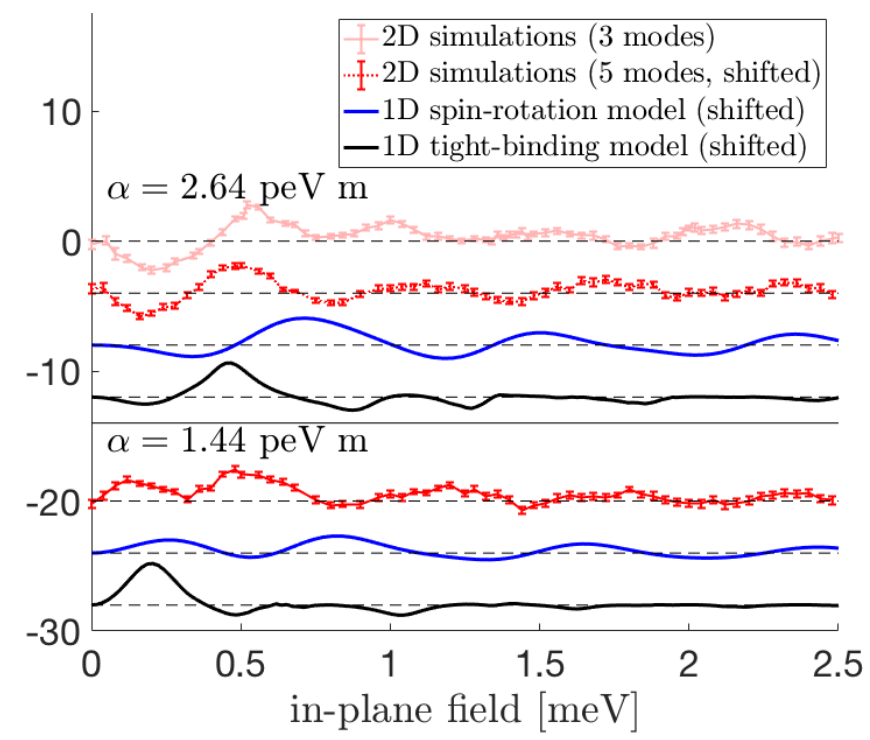

FIG. 6. Zeeman oscillations in resistance anisotropy of a mesoscopic ring calculated with $1 \mathrm{D}$ and $2 \mathrm{D}$ methods at Rashba SO coupling strength $\alpha=2.64 \mathrm{peV}$ m (above) and $\alpha=1.44 \mathrm{peVm}$ (below). Anisotropy (in \%) is calculated between $\varphi_{1}=\pi / 4$ and $\varphi_{2}=3 \pi / 4$ axis from $A_{R}\left(\varphi_{1}, \varphi_{2}\right)$ [Eq. (7)]. 2D simulations were performed for a 3 -mode system (at the higher Rashba field) as well as for a 5 -mode system (both for lower and higher Rashba fields). The isotropic contribution to conductance in the $1 \mathrm{D}$ tight-binding method is fixed to $G_{\mathrm{B}}=5 G_{0}$ (see Appendix A 1). The Dresselhaus SO coupling is fixed at $\beta=0.3 \mathrm{peV} \mathrm{m}$ in all calculations. The mean free path of electrons in the $2 \mathrm{D}$ method is $\ell=2.5 \mu \mathrm{m}$ and the ring radius is $0.61 \mu \mathrm{m}$.

\section{B. Zeeman oscillations in anisotropy}

The oscillations in resistance anisotropy as a function of the Rashba parameter $\alpha$ are correlated with phases acquired by the spins when travelling along the ring, with contributions of both dynamic and geometric origin. Therefore anisotropy oscillations are expected also as a function of the in-plane field strength. Fig. 6 shows Zeeman oscillations in the resistance anisotropy $A_{\mathrm{R}}\left(\varphi_{1}=\pi / 4, \varphi_{2}=3 \pi / 4\right)$ obtained by using the $2 \mathrm{D}$ multi-mode method, the $1 \mathrm{D}$ tight-binding method, and the $1 \mathrm{D}$ spin-rotation model. The oscillations in resistance originate partially from the spin interference between the directly reflected path and the AAS paths (see Appendix C for further discussion). This is modeled by the finite $p_{1}$ term in the $1 \mathrm{D}$ spin-rotation model. To account for disorder effects in the 1D spin-rotation model we choose an amplitude $p_{1}=0.06$ for the directly reflected path and $p_{2}=0.1$ for the AAS paths. A weak Dresselhaus component $\beta=0.3 \mathrm{peV} \mathrm{m}$ is considered in the two cases displayed in the figure, corresponding to Rashba field intensities $\alpha=2.64 \mathrm{peVm}$ and $\alpha=1.44 \mathrm{peV} \mathrm{m}$. We note that all the three methods give oscillation periods of about $0.7 \mathrm{meV}$.

\section{IMPRINTS OF GEOMETRIC PHASE SWITCHING}

In the last years, the relevance of geometric spin phases has been strikingly exemplified in studies on the magnetoconductance of mesoscopic arrays of rings with strong SO coupling.26 This includes the independent manipulation of geometric spin phases by means of weak external in-plane magnetic fields. ${ }^{21}$ Interestingly, a proposal to use an external in-plane field for switching the effective field's topology in SO ring interferometers within the adiabatic regime, causing an observable imprint in the conductance, goes back to a work by Lyanda-Geller $\stackrel{28}{ }$ in the early '90s (see Ref. 29 for a recent observation of Berry phase $e^{30}$ switching in graphene resonators). Still, the adiabatic treatment proposed by Lyanda-Geller appears to be inadequate, which has motivated the search of corresponding effects for nonadiabatic spin dynamics! 31 Recently, this has led to the identification of a so called effective geometric phase ${ }^{32}$ governing a topological transition in the nonadiabatic spin dynamics correlated with a topological transition in the field texture. It has been suggested that this effective phase of geometrical origin could be studied by either interferometric means in ring systems or analyzing resonances in spin or other two-level quantum systems. $\frac{33}{35}$ Here we show that signatures of a transition sharing some of the features associated to the effective geometric phase may be observed in the anisotropy oscillations considered in this work.

$1 \mathrm{D}$ and 2D numerical results show a characteristic shift in the pattern of oscillations of the resistance anisotropy $A_{\mathrm{R}}\left(\varphi_{1}=\pi / 4, \varphi_{2}=3 \pi / 4\right)$ close to the critical line where the in-plane field is equal to the Rashba SO field and the field topology changes ${ }^{32}$ (see panels b, c an $\mathrm{d}$ in Fig. 7). The strength of the Dresselhaus field is weak enough $(\beta=0.3 \mathrm{peV} \mathrm{m})$ to ensure that the total SO field is approximately rotationally symmetric. We note that the resistance calculated from the backscattering probability for AAS paths by using the $1 \mathrm{D}$ spin rotation model $\left(p_{1}=0, p_{2}=0.12\right)$ does not display a clear evidence of a topological transition due to the suppression of Zeeman oscillations (Fig. 7 a).

$1 \mathrm{D}$ results of the spin-rotation model for pure AAS paths are shown in Fig. 7b. In general, the pattern close to the critical line depends on the lead orientation due to nonadiabatic effects and the slight anisotropy of the SO field. However, this effect is not important in our case and it is smoothed out when an average over the lead orientation $\omega$ is performed (data for $\omega=0$ is shown in Appendix D. Motivated by the results shown in Appendix C, we analyze the contribution of directly backscattered electrons in panel c, where $p_{1}=0.06$ is used. In this case, the reflected component interferes with that propagating around the ring and produces an interference pattern with twice the periodicity observed for AAS paths. The interference pattern is thus more complex but, nevertheless, displays the distinct phase dislocation across the critical line. 
$2 \mathrm{D}$ simulations $\sqrt{36}$ in Fig. $7 \mathrm{~d}$ show an agreement with those obtained in the $1 \mathrm{D}$ case with the spin-rotation model in Fig. 7k. It demonstrates that directly reflected spins play a relevant role in the interference pattern. It is worth to note the slightly shorter periodicity as a function of the in-plane field strength in 2D calculations when compared to the $1 \mathrm{D}$ case. This is due to the slightly longer average path around the ring that the electrons experience in the presence of disorder scattering, resulting in a higher Zeeman phase.

\section{CONCLUSIONS}

We have shown that the interplay between Rashba and Dresselhaus [001] SO fields in mesoscopic rings leads to transport anisotropies when probed by an inplane Zeeman field. This is due to the anisotropic nature of the total effective fields that determine the dynamics of (counter)clockwise interfering spin carriers, which also manifests as anisotropic avoiding crossings in the spectrum of isolated rings tuned by the fields. We found that the resistance anisotropy oscillates as a function of both Zeeman and SO field strengths. This suggests that field components can be characterized together with dynamic and geometric spin phases through the measurement of resistance anisotropies.

Our 2D simulations show that the anisotropic signal is expected to be robust against disorder and other sample asymmetries. Resistance anisotropy measurements may therefore provide clear evidence of an effective geometricphase switching in the regime of nonadiabatic spin dynamics, where a direct observation of such topological transition may be challenging due to spin dephasing and relaxation in high Zeeman fields.

\section{ACKNOWLEDGMENTS}

This work was supported by Japan Society for the Promotion of Science through Grant-in-Aid for Scientific Research (C) No. 17K05510, Grant-in-Aid for Specially Promoted Research No. H1505699, Grantin-Aid for Scientific Research on Innovative Areas No. JP15K21717, and by Project No. FIS2014-53385-P (MINECO/FEDER, Spain). JPB thanks the hospitality of Centro Atómico Bariloche (Argentina), where part of this work was done. The 2D simulations were calculated using the Hokusai system provided by Advanced Center for Computing and Communication (ACCC) at RIKEN.
* e-mail: henri.saarikoski@riken.jp

† e-mail: andres.a.reynoso@gmail.com

1 Y. A. Bychkov and E. I. Rashba, J. Phys. C 17, 6039 (1984).

2 G. Dresselhaus, Phys. Rev. 100, 580 (1955).

3 J. Nitta, T. Akazaki, H. Takayanagi, and T. Enoki, Phys. Rev. Lett. 78, 1335 (1997).

4 J. Schliemann, Rev. Mod. Phys. 89, 011001 (2017).

5 J. D. Koralek, C. P. Weber, J. Orenstein, B. A. Bernevig, Shou-Cheng Zhang, S. Mack, and D. D. Awschalom, Nature 458, 610 (2009)

6 B. A. Bernevig, J. Orenstein, and S.-C. Zhang, Phys. Rev. Lett. 97, 236601 (2006).

7 M. Kohda, V. Lechner, Y. Kunihashi, T. Dollinger, P. Olbrich, C. Schönhuber, I. Caspers, V. V. Bel'kov, L. E. Golub, D. Weiss, K. Richter, J. Nitta, and S. D. Ganichev, Phys. Rev. B 86, 081306(R) (2012).

8 J. Schliemann, J. C. Egues, and D. Loss, Physical Review Letters 90 (14), 146801 (2003).

9 S. D. Ganichev, V. V. Belkov, L. E. Golub, E. L. Ivchenko, Petra Schneider, S. Giglberger, J. Eroms, J. De Boeck, G. Borghs, W. Wegscheider, D. Weiss, and W. Prettl, Phys. Rev. Lett. 92, 256601 (2004).

10 A. Sasaki, S. Nonaka, Y. Kunihashi, M. Kohda, T. Bauernfeind, T. Dollinger, K. Richter und J. Nitta, Nature Nanotechnology 9, 703 (2014).

11 J. Schliemann and D. Loss, Phys. Rev. B 68, 165311 (2003).

12 J. B. Miller, D. M. Zumbühl, C. M. Marcus, Y. B. LyandaGeller, D. Goldhaber-Gordon, K. Campman, and A. C. Gossard, Phys. Rev. Lett. 90, 076807 (2003).
13 A. G. Malshukov, V. A. Froltsov, and K. A. Chao, Phys. Rev. B 59, 5702 (1999).

14 Y. Aharonov and A. Casher, Phys. Rev. Lett. 53, 319 (1984).

15 B.L. Altshuler, A.G. Aronov, and B.Z. Spivak, Pisma Zh. Eksp. Teor. Fiz. 33, 101 (1981) [JETP Lett. 33, 94 (1981)].

16 M. I. Dyakonov and V. Y. Kachorovskii, Sov. Phys. Semicond. 20, 110 (1986).

17 S. V. Iordanskii, Y. B. Lyanda-Geller, and G. E. Pikus, JETP Lett. 60, 206 (1994).

18 R. Winkler, Spin-Orbit Coupling Effects in TwoDimensional Electron and Hole Systems, Springer Tracts in Modern Physics 191 (Springer, New York) (2003).

19 C. W. Groth, M. Wimmer, A. R. Akhmerov, and X. Waintal, New J. Phys 16, 063065 (2014).

20 T. Ando, Phys. Rev. B 44, 8017 (1991).

21 F. Nagasawa, D. Frustaglia, H. Saarikoski, K. Richter, and J. Nitta, Nature Comm. 4, 2526 (2013).

22 K. Richter, Physics 5, 22 (2012).

23 C. Ortix, Phys. Rev. B 91, 245412 (2015).

24 Z.-J. Ying, P. Gentile, C. Ortix, and M. Cuoco, Phys. Rev. B 94, 081406(R) (2016).

25 In the absence of Dresselhaus SO interaction, the total average field acting on a spin in a round-trip is isotropic with respect to the in-plain field direction. Still, the resistance across the system is weakly anisotropic with respect to the orientation of the lead relative to that of the in-plane field. We discuss this effect in Appendix B

${ }^{26}$ F. Nagasawa, J. Takagi, Y. Kunihashi, M. Kohda, and J. Nitta, Phys. Rev. Lett. 108, 086801 (2012). 

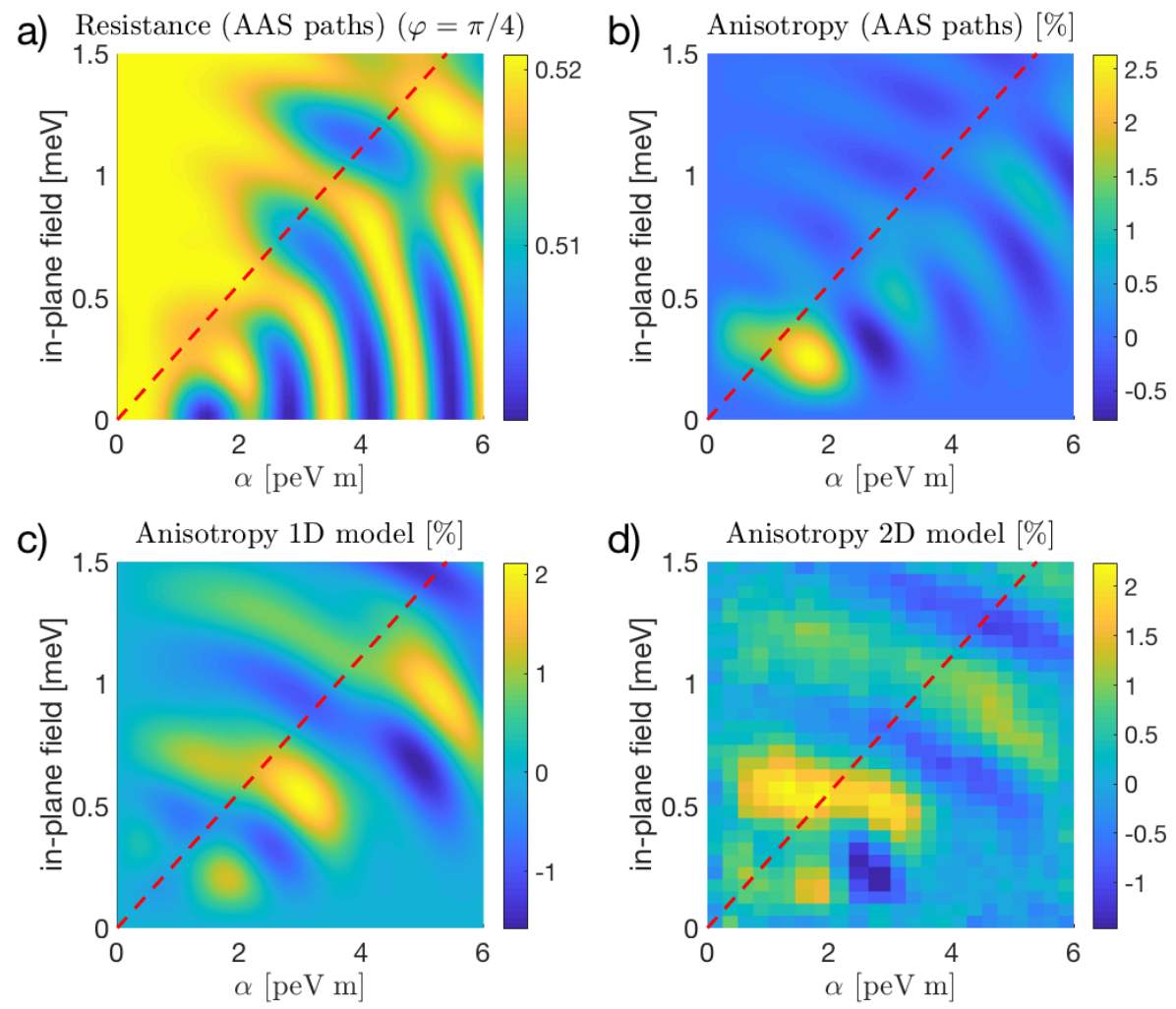

FIG. 7. a) Resistance calculated from the backscattered AAS paths (1D spin-rotation model with $p_{1}=0, p_{2}=0.12$ ) for an in-plane field angle $\varphi_{1}=\pi / 4$. b) Resistance anisotropy for AAS paths averaged over lead orientation $\omega$ in the 1D spin-rotation model. Note the phase shift in the anisotropy oscillations near the critical line (dashed) where the Rashba SO field strength is equal to the in-plane field strength. c) Resistance anisotropy calculated using the 1D spin-rotation model with a direct backscattering amplitude $p_{1}=0.06$ added. d) Resistance anisotropy calculated using the $2 \mathrm{D}$ model for mean-free path of $\ell=2.5 \mu \mathrm{m}$ and $\omega=0$. Dresselhaus SO $\beta=0.3 \mathrm{peV} \mathrm{m}$ in all calculations. All anisotropies are calculated between in-plane field directions $\varphi_{1}=\pi / 4$ and $\varphi_{2}=3 \pi / 4$ using $A_{\mathrm{R}}\left(\varphi_{1}, \varphi_{2}\right)$ [Eq. [7]].

27 D. Frustaglia and K. Richter, Phys. Rev. B 69, 235310 (2004).

28 Y. Lyanda-Geller, Phys. Rev. Lett. 71, 657 (1993).

${ }^{29}$ F. Ghahari et al., Science 356, 845849 (2017).

30 M. V. Berry, Proc. R. Soc. London, Ser. A 392, 45 (1984).

31 Y. Aharonov and J. Anandan, Phys. Rev. Lett. 58, 1593 (1987).

${ }^{32}$ H. Saarikoski, J. E. Vázquez-Lozano, J. P. Baltanás, F. Nagasawa, J. Nitta, and D. Frustaglia, Phys. Rev. B 91 241406(R) (2015).

33 H. Saarikoski, J. P. Baltanás, J. E. Vázquez-Lozano, J. Nitta, and D. Frustaglia, J. Phys. Cond. Mat. 28, 166002 (2016).

34 A. A. Reynoso, J. P. Baltanás, H. Saarikoski, J. E. Vázquez-Lozano, J. Nitta, and D. Frustaglia, New J. Phys. 19, 063010 (2017).

35 J. P. Baltanás, H. Saarikoski, A. A. Reynoso, and D. Frustaglia, Phys. Rev. B 96, 035312 (2017).

36 Smoothing has been performed on the data to reduce noise associated with the lattice disorder model. The raw data is shown in Appendix D

37 F. E. Meijer, A. F. Morpurgo, T. M. Klapwijk, T. Koga, and J. Nitta, Phys. Rev. B 70, 201307(R) (2004)

Appendix A: Computational methods

\section{1D tight-binding approach}

The 1D tight-binding method is based on a single channel approach where we apply the customary finite difference method to the Hamiltonian of Eq. (5). The ring is reduced to $N$ sites separated by the lattice spacing $a_{0}=$ $P / N$, with $P=2 \pi R_{c}$ the ring perimeter, and site positions $\mathbf{r}_{n}=\left(R_{c} \cos (2 \pi(n-1) / N), R_{c} \sin (2 \pi(n-1) / N)\right)$, where $\mathbf{r}_{N+1}=\mathbf{r}_{1}$ in ring geometry. The tight-binding Hamiltonian of the ring becomes 


$$
\begin{aligned}
\hat{H} & =\sum_{n=1}^{N} \sum_{\sigma \sigma^{\prime}}\left[\hat{c}_{\mathbf{r}_{n} \sigma}^{\dagger}(S)_{\sigma \sigma^{\prime}} \hat{c}_{\mathbf{r}_{n} \sigma^{\prime}}+\left(\hat{c}_{\mathbf{r}_{n} \sigma}^{\dagger}\left(T_{n}\right)_{\sigma \sigma^{\prime}} \hat{c}_{\mathbf{r}_{n+1} \sigma^{\prime}}+h . c .\right)\right] \\
S & =\frac{\hbar^{2}}{m^{*} a_{0}^{2}} \sigma_{0}+E_{Z}\left(\cos \phi \sigma_{x}+\sin \phi \sigma_{y}\right) \\
T_{n} & =-\frac{\hbar^{2}}{2 m^{*} a_{0}^{2}} \sigma_{0}+\frac{i}{2 a_{0}} \frac{\mathbf{r}_{n+1}-\mathbf{r}_{n}}{\left|\mathbf{r}_{n+1}-\mathbf{r}_{n}\right|} \cdot\left(\beta \sigma_{x}+\alpha \sigma_{y},-\beta \sigma_{y}-\alpha \sigma_{x}\right)
\end{aligned}
$$

where the $\sigma$ and $\sigma^{\prime}$ summations run on $\{\uparrow, \downarrow\}$, the up and down spin projections along $z$-axis. The spin independent nearest neighbor hopping in the model is $t_{h}=\hbar^{2} /\left(2 m^{*} a_{0}^{2}\right)$.

By choosing $a_{0} \approx \lambda_{F} / 10$ we ensure parabolic behavior at the working energy. The left and right leads are connected to site $\mathbf{r}_{L}\left(=\mathbf{r}_{N / 2}\right)$ and site $\mathbf{r}_{R}\left(=\mathbf{r}_{1}\right)$, respectively. We choose both leads well coupled to the ring. This is achieved with the leads being simple tightbinding chains with large bandwidth hopping $t_{c} \gg t_{h}$. The Green function at the end site of each semi-infinite chain lead is $g_{c}^{r}(E) \approx-i \sigma_{0} /\left|t_{c}\right|$, its real part being negligible since we work at the center of the band assuring featureless energy dependence. The self-energy of leads $K=\{L, R\}$ are $\hat{\Sigma}_{K}^{r}(E) \approx-i\left|\mathbf{r}_{K}\right\rangle\left\langle\mathbf{r}_{K}\left|\sigma_{0} t_{i}^{2} /\right| t_{c}\right|$ with the intermediate hopping $t_{i}$ fixed at the optimum $t_{i}=\left(t_{h}+t_{c}\right) / 2$. The conductance is computed using the retarded and advanced Green functions $\hat{G}^{r}(E)=$ $\left(\hat{G}^{a}(E)\right)^{\dagger}=\left[\left(E+i 0^{+}\right) I_{N}-\hat{H}-\hat{\Sigma}_{L}^{r}-\hat{\Sigma}_{R}^{r}\right]^{-1}$ with

$$
G=\frac{e^{2}}{h} \operatorname{Tr}\left[\hat{\Gamma}_{R}\left(E_{F}\right) \hat{G}^{r}\left(E_{F}\right) \hat{\Gamma}_{L}\left(E_{F}\right) \hat{G}^{a}\left(E_{F}\right)\right],
$$

where the level-width function of lead $K$ is given by $\hat{\Gamma}_{K}(E)=i\left(\hat{\Sigma}_{K}^{r}(E)-\hat{\Sigma}_{K}^{a}(E)\right)$.

The 1D tight-binding method does not take into account the large spin-dephased contribution that appear in 2D multi-mode calculations with disorder. When comparing with other methods we add therefore a large isotropic background conductance $G_{\mathrm{B}}$ to the conductance that is independent of $\varphi$ as $G_{\Omega}(\varphi)=G_{1 \mathrm{D}, \Omega}(\varphi)+G_{\mathrm{B}}$.

\section{1D spin-rotation method}

We use a 1D single-mode approach to study spininterference by introducing the effective spin Hamiltonian

$$
H_{\mathrm{S}}(\theta)=\left(\mathbf{B}_{\mathrm{R}}+\mathbf{B}_{\mathrm{D}}+g^{*} \mu_{\mathrm{B}} \mathbf{B}_{\text {in }- \text { plane }}\right) \cdot \boldsymbol{\sigma},
$$

where the effective Rashba and Dresselhaus SO fields acting on a spin moving at constant speed are given by

$$
\begin{gathered}
\mathbf{B}_{\mathrm{R}}=B_{\mathrm{R}}(\sin \theta \hat{\mathbf{x}}-\cos \theta \hat{\mathbf{y}}), \\
\mathbf{B}_{\mathrm{D}}=B_{\mathrm{D}}(-\cos \theta \hat{\mathbf{x}}+\sin \theta \hat{\mathbf{y}}),
\end{gathered}
$$

respectively. Here $B_{\mathrm{R}}=k_{\|} \alpha$ and $B_{\mathrm{D}}=k_{\|} \beta, k_{\|}$is the wave number along the direction of propagation, and $\theta$ is the direction of the $\mathbf{k}$-vector with $\mathbf{k}=k_{\|}(\cos \theta \hat{\mathbf{x}}+\sin \theta \hat{\mathbf{y}})$. Disorder in realistic rings suppresses multiple windings of spin around the ring and therefore we assume that AAS paths are the most important ones (Fig. 1). As a consequence, resistance is determined by backscattering of spins.

Using Eq. (6), the backscattering probability amplitude is calculated by adding the contributions of the reflected wave at the intersection and the waves transmitted around the ring. The conductance is then given by

$$
G=\left(2-p_{r}\right) G_{0}=\left(2-|\psi|^{2}\right) G_{0}
$$

where is $p_{r}=|\psi|^{2}$ is the backscattering probability. In pure 1D models, reflected waves at the intersections may acquire a phase shift of $\pi$. This is associated with a negative value of $p_{1}$ in Eq. (6). In $2 \mathrm{D}$ models, modemixing at the intersections affects also phases of the waves transmitted around the ring. In addition, disorder is assumed to give rise to spin dephasing and relaxation. Therefore we keep $p_{1,2}$ as free parameters in the 1D spin-rotation model. At the intersection, a spin can move either back to the lead or go around the ring in the clockwise/anticlockwise direction. From this we can derive that $p_{2}<\sqrt{\left(1-p_{1}^{2}\right) / 2}$ in general.

The spin evolution in a clockwise rotating round-trip around the ring is calculated from the unitary spin propagator

$$
C=\prod_{m=1}^{n} \exp \left(i H_{\mathrm{S}}\left(\theta_{m}\right) \Delta t\right)
$$

where a full rotation $(\theta=\pi / 2 \rightarrow-3 \pi / 2$ for the clockwise path in Fig. 11 is divided into $n$ steps of time interval $\Delta t$ and $\theta_{m}=\pi / 2-2 \pi m / n$. The anticlockwise rotating round-trip operator $A$ is obtained likewise by calculating the rotation in opposite direction $(\theta=-\pi / 2 \rightarrow 3 \pi / 2$ for the anticlockwise path in Fig. 11). Details of the method are described in Ref. 33. From Eqs. (6) and (A8) we get a final expression for conductance of one spin-compensated incoming mode

$$
\begin{gathered}
G=\left[2-2 p_{1}^{2}+2 p_{1}^{2} p_{2}^{2} \operatorname{Tr}\left(C+C^{\dagger}+A+A^{\dagger}\right)\right. \\
\left.+p_{2}^{2}\left(4+\operatorname{Tr}\left(A^{\dagger} C+C^{\dagger} A\right)\right)\right] G_{0} .
\end{gathered}
$$




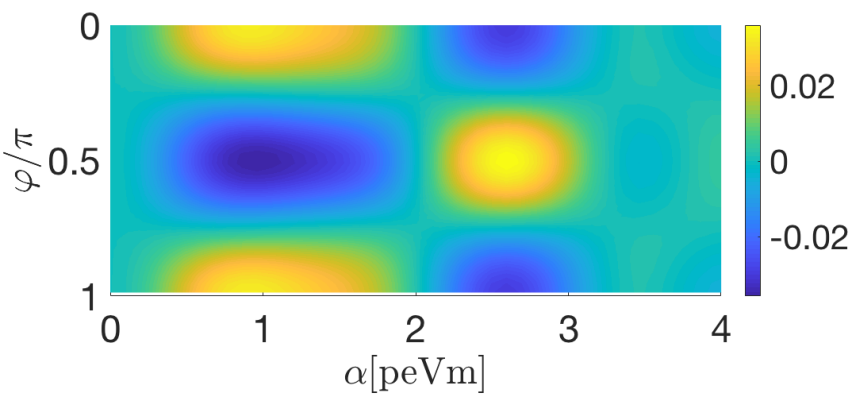

FIG. 8. Resistance anisotropy Eq. (8) (in \%) in isotropic Rashba spin-orbit field and vanishing Dresselhaus field $\beta=$ $0 \mathrm{peVm}$. The figure is calculated for the backscattered AAS paths $\left(p_{1}=0, p_{2}=0.035\right)$ with the $1 \mathrm{D}$ spin-rotation model as a function of in-plane field direction $\varphi$ and Rashba spin-orbit field $\alpha$. Maximum anisotropy occurs between in-plane field axis $\varphi=0$ and $\varphi=\pi / 2$.

\section{Appendix B: Resistance anisotropy in isotropic spin-orbit fields (case $\beta=0$ )}

As discussed in the main text, resistance anisotropy in our calculations arises mostly due to spin-orbit field anisotropy. In the absence of the Dresselhaus SO interaction, the rotation symmetry of the Rashba SO field implies that the total average field acting on a spin in a round-trip around the ring does not depend on the in-plane field direction, so that resistance anisotropy is not expected. Still, our calculations exhibit a small but nonvanishing resistance anisotropy in isotropic spin-orbit fields as a function of the relative orientation of the leads and the external in-plane magnetic field, as we show below.

Figure 8 shows resistance anisotropy calculated from the AAS backscattering probability in a Rashba field using the $1 \mathrm{D}$ spin-rotation model. The results show that at $\beta=0$ the resistance anisotropy is largest between inplane fields parallel $(\varphi=0)$ and normal $(\varphi=\pi / 2)$ to the lead direction, and vanishes between directions $\varphi=\pi / 4$ and $3 \pi / 4$.

Due to isotropic character of the Rashba SO field, the resistance anisotropy at $\beta=0$ is presumably more sensitive to effects of disorder. Indeed, $2 \mathrm{D}$ calculations in 5 -mode disordered systems show only a weak resistance anisotropy as a function of the in-plane field direction (see Fig. 9). These oscillations in resistance anisotropy are in close agreement with the results obtained by using the $1 \mathrm{D}$ spin-rotation model for AAS paths $\left(p_{1}=0\right.$, $p_{2}=0.035$ shown in Fig. 9p.

\section{Appendix C: Zeeman oscillations in resistance}

The anisotropy oscillations as a function of the inplane field are related to Zeeman oscillations in the resistance associated with the dynamic phase that the spins

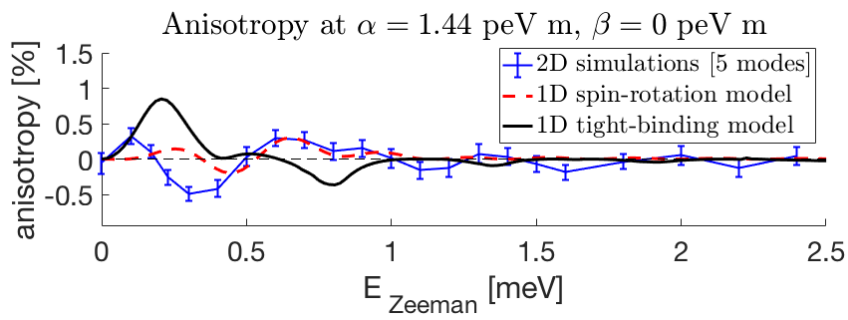

Anisotropy at $\alpha=2.64 \mathrm{peV} \mathrm{m}, \beta=0 \mathrm{peV} \mathrm{m}$

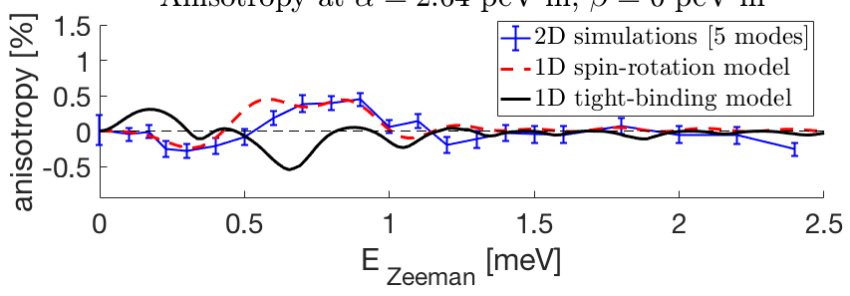

FIG. 9. Weak oscillations in resistance anisotropy of a $R=$ $610 \mathrm{~nm}$ ring system as a function of in-plane field strength for isotropic Rashba spin-orbit fields $\alpha=1.44 \mathrm{peVm}$ (above) and $\alpha=2.64$ peVm (below) in the absence of Dresselhaus coupling $(\beta=0)$. Resistance anisotropies are calculated between $\varphi=0$ and $\varphi=\pi / 2$ axis using Eq. 7). Results are calculated using the $2 \mathrm{D}$ method in a $W=68 \mathrm{~nm}$ wide wire with 5 transport modes and compared to the $1 \mathrm{D}$ spin-rotation model. The 1D model assumes weak backscattering via AAS paths only $\left(p_{1}=0, p_{2}=0.035\right)$.

accumulate in a round-trip around the ring. These oscillations are especially prominent in loop geometries 32 Since the dynamic phases acquired for clockwise and counterclockwise rotating paths in symmetric ring geometries are equal, Zeeman oscillations do not appear for directly transmitted (Aharonov-Casher) paths in the absence of spin-orbit interaction. However, Zeeman oscillations in resistance appear due to interference between directly reflected and (counter)clockwise propagating paths. Fig. 10 shows resistance calculated for AAS paths with the 1D spin-rotation model and 2D simulations. The Dresselhaus term is fixed here at $\beta=$ $0.3 \mathrm{peV} \mathrm{m}$. The Zeeman oscillation period is about 0.6 $\mathrm{meV}$ in the $1 \mathrm{D}$ model corresponding to the Zeeman phase of $2 \pi$ in a round-trip around the $610 \mathrm{~nm}$ radius ring.

\section{Appendix D: Supplementary data}

We present here supplementary data associated with the topological transition in resistance anisotropy.

Figure 11a shows the topological transition at a fixed lead direction $\omega=0$. We note that lead direction averaging (Fig. 7b) evens out nonadiabatic effects close to the critical line leaving a slightly more clear signature of the topological transition. Figure $11 \mathrm{~b}$ presents raw data of the 2D model associated with Fig. $7 \mathrm{~d}$. Noise in the data is due to lattice disorder model.

Finally, we note that the resistance anisotropy persists when the disorder density is increased from the value 


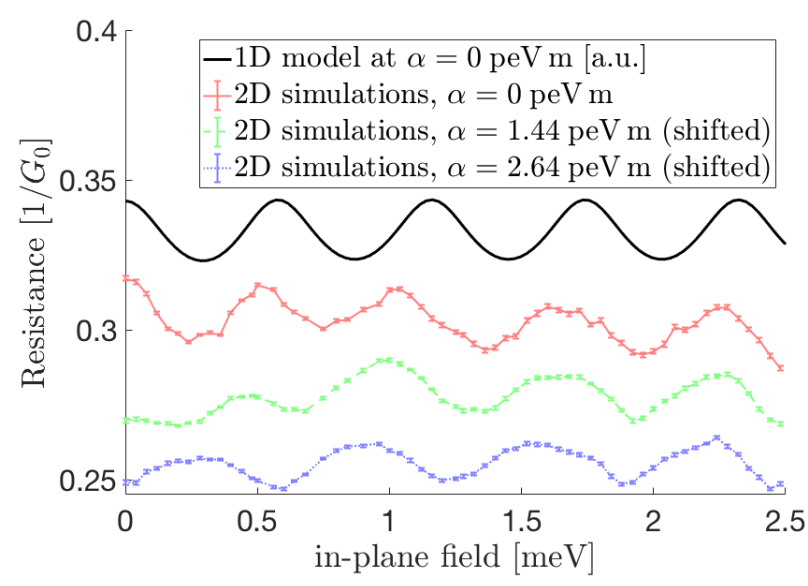

FIG. 10. Zeeman oscillations in resistance of a 5-mode mesoscopic ring system calculated with the 2D method at Rashba SO coupling strengths $\alpha=0 \mathrm{peVm}, \alpha=1.44 \mathrm{peVm}$, and $\alpha=2.64 \mathrm{peV} \mathrm{m}$. The results are compared to the single-mode $1 \mathrm{D}$ spin-rotation model at $\alpha=0 \mathrm{peV} \mathrm{m}$ using $p_{1}=0.06$ and $p_{2}=0.1$ (thick line). The Dresselhaus SO coupling is fixed at $\beta=0.3 \mathrm{peVm}$ in all calculations. The mean free path of electrons in the $2 \mathrm{D}$ simulations is $\ell=2.5 \mu \mathrm{m}$, the ring radius is $0.61 \mu \mathrm{m}$, and the in-plane magnetic field angle $\varphi=3 \pi / 4$. The oscillations indicate interference between waves reflected at the intersection and waves transmitted around the ring.

used in the main text (corresponding to $\ell=2.5 \mu \mathrm{m}$ ). Figure 11. shows the anisotropy $A_{\mathrm{R}}(\varphi=\pi / 4, \varphi=3 \pi / 4)$ for $\beta=0.3 \mathrm{peV} \mathrm{m}$ and in-plane field strength of 0.17 $\mathrm{meV}$, i.e. the same as values used in Fig. 3 and Fig. 4 , and 5 transport modes. Resistance anisotropy increases slightly with decreasing electron mean free path. In the clean regime, $\ell \gg 3 \mu \mathrm{m}$, direct paths through the system dominate over AAS paths and resistance anisotropy decreases.

Figure 11: shows that the sign of $A_{\mathrm{R}}(\varphi=\pi / 4, \varphi=$ $3 \pi / 4)$ reverses in the higher Rashba SO coupling strength $\alpha=2.64 \mathrm{peVm}$ at moderate disorder densities with $0.45 \mu \mathrm{m}<\ell<3.0 \mu \mathrm{m}$. This is consistent with AAS path interference effects described in the main text. However, we observe no sign reversal in the highly disordered regime $\ell<0.15 \mu \mathrm{m}$. Our calculations show that in this highly disordered regime, resistance anisotropy originates mainly from interference effects within the multi-mode wire in contrast to AAS ring interference. This is demonstrated by transport simulations in a straight 5 -mode wire without a ring structure in Fig. $11 \mathrm{~d}$. In the 5-mode wire resistance anisotropy is not significant in the regime of moderate disorder but increases with disorder strength when $\ell<0.45 \mu \mathrm{m}$. Moreover, anisotropy does not reverse sign at higher Rashba SO coupling $\alpha=2.64 \mathrm{peV} \mathrm{m}$. This pattern can be directly compared to calculations in a ring structure in Fig. 11. We conclude that AAS ring interference is the most important source of resistance anisotropy in the ring device in the regime of moderate disorder. 
a)

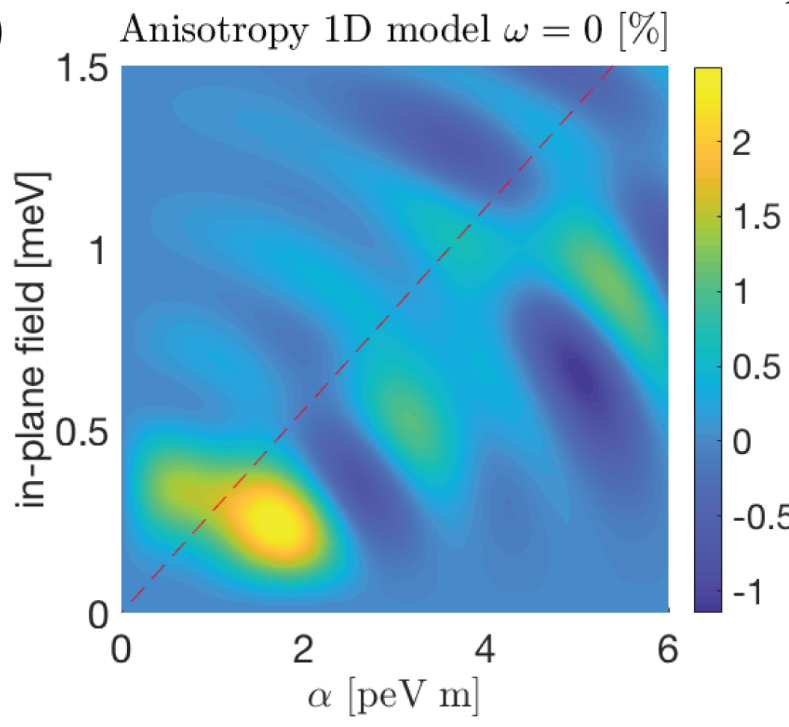

c)

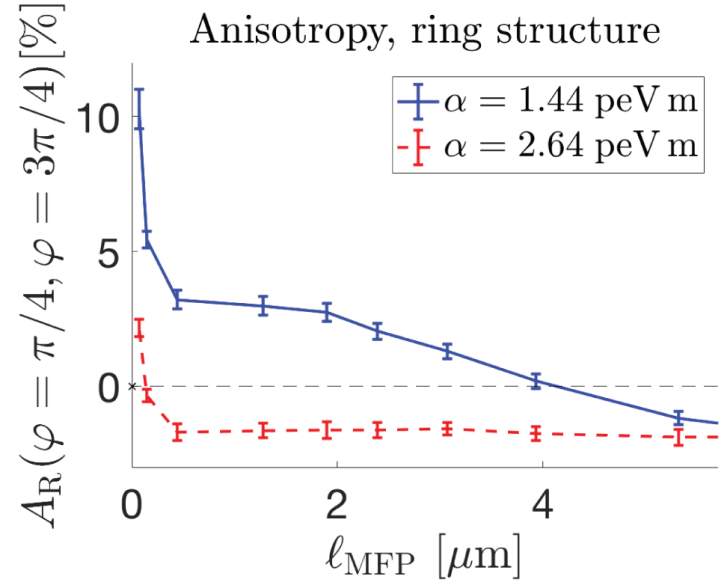

b)

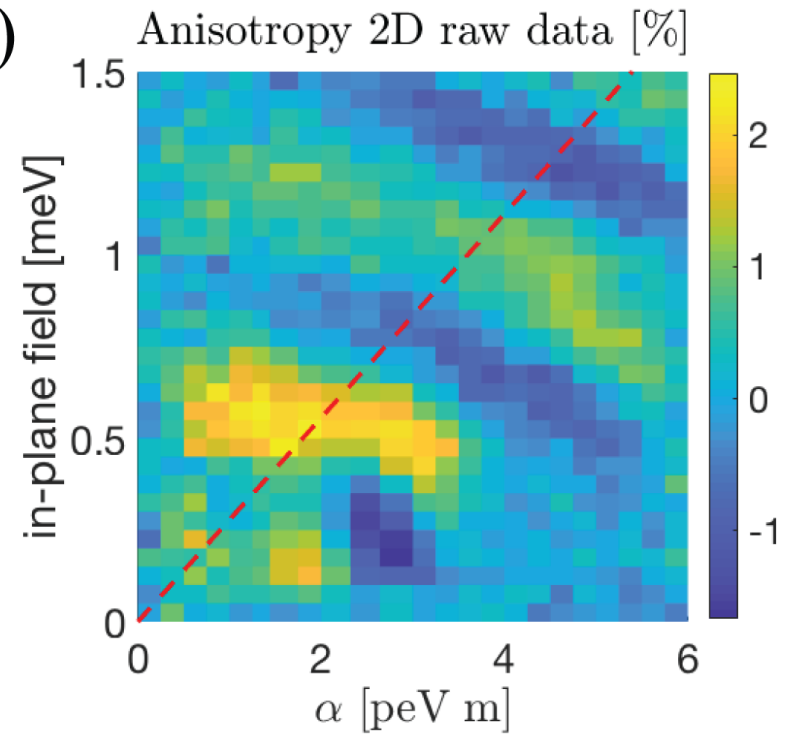

d)

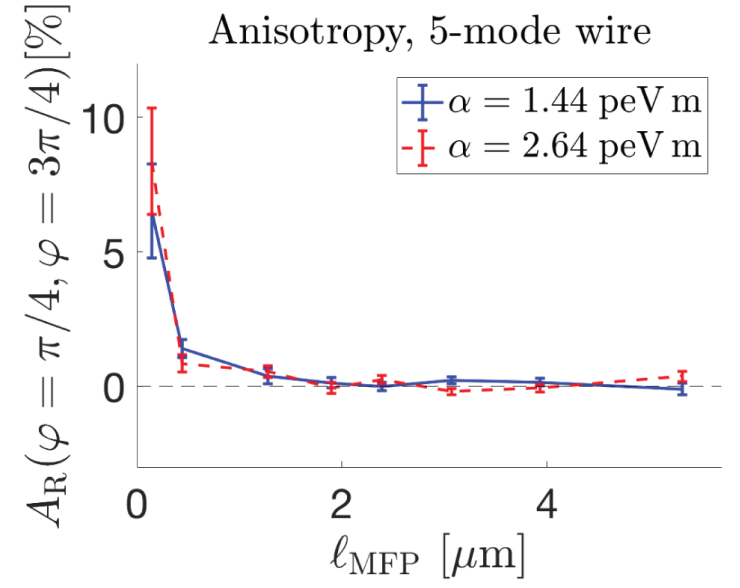

FIG. 11. a) Resistance anisotropy calculated from the backscattering probability for AAS paths using the 1D spin-rotation model at fixed lead orientation along $x$-direction $(\omega=0)$. b) Raw data of resistance anisotropy calculated using the 2D model with mean-free path of electrons $\ell=2.5 \mu \mathrm{m}$ and $\omega=0$. All anisotropies are calculated between in-plane field directions $\varphi_{1}=\pi / 4$ and $\varphi_{2}=3 \pi / 4$ and with Dresselhaus SO strength $\beta=0.3 \mathrm{peV} \mathrm{m}$ using Eq. (7). The dashed line in the figures shows the critical line where the Rashba SO field strength is equal to the in-plane field strength. c) Resistance anisotropy between $\varphi=\pi / 4$ and $\varphi=3 \pi / 4$ axis calculated as a function of electron mean free path $\ell$. Here $\beta=0.3$ peV m and in-plane field strength of $0.17 \mathrm{meV}$. The results are obtained using the $2 \mathrm{D}$ method with 5 transport modes in the ring. d) Resistance anisotropy between $\varphi=\pi / 4$ and $\varphi=3 \pi / 4$ axis in a straight 5 -mode wire (without the ring structure). The Dresselhaus SO strength and the Zeeman strength are the same as in c). Anisotropy vanishes in the regimes of moderate and low disorder. 